\title{
Effect of Eight Weelss of Continuous Exercise and Resveratrol Supplementation on Insulin Resistance and Pyruvate Dehydrogenase Kinase 4 (PDI4) Expression in the Soleus Muscle of Elderly Diabetic Rats
}

Ali Salehi (PhD Candidate)

Department of Sport Physiology, Sari Branch, Islamic Azad University, Sari, Iran

Hajar Abbaszadeh (PhD)

Department of Sport Physiology, Sari Branch, Islamic Azad University, Sari, Iran

Parvin Farzanegi (PhD)

Department of Sport Physiology, Sari Branch, Islamic Azad University, Sari, Iran

Corresponding author: Hajar Abbaszadeh

Tel: +989112163511

Email:h.abaszade61@gmail.com

Address: Department of Sport

Physiology, Sari Branch, Islamic Azad University, Sari, Iran

Received: 18 Dec 2018

Revised: 08 Jan 2019

Accepted: 09 Jan 2019

\section{c) (i) (8)}

This work is licensed under a Creative Commons Attribution 4.0 License.
ABSTRACT

Background and objectives: Pyruvate dehydrogenase kinase 4 (PDK4) is an enzyme involved in the regulation of glucose and fatty acid metabolism and homeostasis. The purpose of this study was to investigate effects of continuous exercise and resveratrol supplementation on insulin resistance and expression of PDK4 in the soleus muscle of elderly rats with type 2 diabetes.

Methods: After inducing diabetes in 42 old male rats (mean weigh: 250-300 g, aged about two years), the rats were randomly divided into six groups: healthy control, diabetic control, continuous exercise, supplementation, continuous exercise + supplementation and sham. The continuous exercise group was subjected to eight weeks of running, five sessions a week. Daily $20 \mathrm{mg} / \mathrm{Kg}$ body weight resveratrol supplementation was administered to the supplementation and supplementation + exercise groups. To evaluate insulin resistance, the H0MA-IR insulin resistance index was calculated as the product of insulin $(\mu \mathrm{Il} / \mathrm{mL})$ and glucose (mmol/L) divided by 22.5. Expression of the PDK4 gene in the soleus muscle was analyzed by RT-PCR. Data were analyzed with IBMI SPSS Statistics 23 using one-way ANOVA and Tukey's post hoc test at a significance level of 0.05 .

Results: Resveratrol alone or combined with continuous exercise significantly increased PIJK4 expression and slightly decreased insulin resistance in the subjects.

Conclusion: 0ur results show that diabetes is associated with reduced PDK4 expression and increased insulin resistance. These effects can be effectively reversed in the soleus muscle of elderly diabetic rats by resveratrol supplementation alone or combined with continuous exercise.

Keywords: Pyruvate Dehydrogenase Kinase, Insulin Resistance, Exercise, Resveratrol, Type 2 Diabetes.

This paper should be cited as: Salehi A, Abbaszadeh H, Farzanegi P[Effect of Eight Weeks of Continuous Exercise and Resveratrol Supplementation on Insulin Resistance and Pyruvate Dehydrogenase Kinase 4 (PDK4) Expression in the Soleus Muscle of Elderly Diabetic Rats]. mljgoums. 2019; 13(4):8-13 


\section{INTRODUCTION}

Aging is a natural process that increases risk of debility and mortality. In this process, multiple cellular and molecular impairments ultimately lead to chronic illness. Although aging is not a disease, it is a risk factor for most chronic diseases (1). Changes related to aging and inactivity as well as changes in the body composition are known causes of metabolic diseases and diabetes (2). Type 2 diabetes is the result of complex interactions between genetic and environmental factors that affect fat and glucose metabolism (3-5). Caring for diabetics is a complex issue that requires a series of interventions to improve glycemic control. In this regard, physical activity and exercise have long been considered as key pillars of diabetes care and management, mainly due to their low cost and non-pharmacological nature. Physical activity can help prevent or treat various metabolic disorders by improving blood glucose and lipid levels (6). It is well demonstrated that exercise can improve glucose uptake in the skeletal muscles of diabetic patients by increasing the activity of AMP kinases (7-9). Pyruvate dehydrogenase lipoamide kinase isozyme 4 (PDK4) is a key enzyme involved in the regulation of glucose and fatty acid metabolism and homeostasis $(10,11)$. The enzyme is found in the pancreatic islets, heart and skeletal muscles, which have high glucose demand and high rates of fatty acids oxidation $(12,13)$. PDK4 is overexpressed during fasting, exercise, and in diabetic individuals. PDK4 constantly reduces carbohydrate oxidation and glucose and glycogen use during exercise $(2,7)$. In fact, PDK4 expression in the skeletal muscles increases in response to all exercise protocols, especially endurance training. Wang et al. found that PDK4 expression after resistance training and symmetrical endurance training is 2.2 times higher than after a single session of endurance training (14). Type 2 diabetes induces hepatic and peripheral insulin resistance, and during moderate-intensity aerobic exercise, peripheral blood glucose uptake is more than the amount of glucose produced by the liver, which leads to a decrease in blood glucose level. Longterm exercise reduces plasma insulin level, which in turn lowers the risk of hypoglycemia in type 2 diabetes (15-18). Nowadays, much attention has been given to management of diabetes through non-pharmaceutical strategies such as exercise and herbal supplements (19, 20). Resveratrol is a natural polyphenol produced in response to pathogens and environmental stress in purple and reddish plants (21-23). Research has shown that resveratrol has anti-inflammatory, antioxidant and anti-diabetic effects $(24,25)$. Thomas et al. revealed that resveratrol exerts anti-diabetic effects by altering PDK4 expression via the PGC-1 $\alpha$ pathway in skeletal muscles of elderly diabetic patients (26). In this study, we aimed to examine effects of continuous exercise with or without resveratrol supplementation on PDK4 expression in the soleus muscle and insulin resistance of elderly type 2 diabetic rats.

\section{MATERIALS AND METHODS}

This experimental study was approved by the animal care and use committee at the Islamic Azad University of Sari (approval reference number: R.IAU.SARI.REC.1397.8). Forty-two male elderly Wistar rats (age: 40-50 weeks, weight: $250-300 \mathrm{~g}$ ) which were replicated at the Azad University of Sari Research Center, were randomly divided into six groups (7 rats in each group): normal control, diabetic control, continuous exercise, supplementation, continuous exercise+supplementation and sham. Subjects were kept in transparent polycarbonate cages (dimensions: 30 x 15 x 15 cm, manufactured by Razi Rad Co.) at ambient temperature of $20 \pm 2{ }^{\circ} \mathrm{C}$, humidity of $50 \% \pm 5$ and 12:12 light dark cycle. The subjects had access to food and water ad libitum. To minimize stress, rats in the training groups were familiarized with a rodent treadmill for five consecutive days (with a speed of 10 $\mathrm{m} / \mathrm{min}$ at $0 \%$ inclination for $5 \mathrm{~min} /$ day) (27, 28). Rats in the continuous exercise group were subjected to eight weeks of running, five days a week. The exercise intensity (running speed) was set at $15 \mathrm{~m} / \mathrm{min}$ for 5 minutes in the initial week. The speed and time of training were gradually increased every week by $1-2 \mathrm{~m} / \mathrm{min}$ and $1-2$ minutes, respectively. Eventually, the running speed reached 28 $\mathrm{m} / \mathrm{min}$ in the last week (29). Diabetes was induced by intraperitoneal injection of 50 $\mathrm{mg} / \mathrm{Kg}$ body weight streptozotocin. Diabetes was confirmed by checking blood samples from the corner of the eye and fasting glucose level of $>250 \mathrm{mg} / \mathrm{dl}$ (30). In the supplementation and supplementation + 
exercise groups, $20 \mathrm{mg} / \mathrm{Kg}$ body weight resveratrol was intraperitoneally injected daily $(24,30,31)$. Ketamine and xylocaine was intraperitoneally injected to the subjects 48 hours after the last exercise session and 10-12 hours of fasting. In addition to blood sampling, tissue samples were taken from the soleus muscle and stored at $-80{ }^{\circ} \mathrm{C}$. Plasma glucose concentration was measured by a colorimetric method based on enzymatic reaction of glucose oxidase using commercial kits (Pars Azmun Co., Iran). Plasma insulin was measured using ELISA kits. To evaluate insulin resistance, the HOMA-IR (Homeostasis Model Assessment-Insulin Resistance) was calculated as the product of insulin $(\mu \mathrm{IU} / \mathrm{mL})$ and glucose $(\mathrm{mmol} / \mathrm{L})$ divided by 22.5. Expression of the PDK4 gene in the soleus muscle was analyzed by RTPCR. First, the tissue samples were homogenized in phosphate buffer ( $\mathrm{pH}$ 7.0) at $4{ }^{\circ} \mathrm{C}$ with a homogenizer. Total RNA was extracted from the samples using the RNXPlus kit (SinaClon; RN7713C). Nanodrop ND1000 spectrophotometer (Thermo Sci., Newington, NH) was used to estimate the quantity and quality of the extracted RNA. Synthesis of cDNA was done using the Revert Aid Reverse Transcriptase (Thermo science, Germany) at $42{ }^{\circ} \mathrm{C}$ for 1 hour (Thermo science, Germany). A Rotor Gene 6000 (Corbett Research, Australia) thermocycler and Real Q-PCR 29 Master Mix Kit (Amplicon, Denmark) in 40 cycles were applied for amplifications. Each reaction solution contained $5 \mu 1$ of master mix and 100 $\mathrm{nm}$ of primers. Primer sequences were as follows:

PDK4, GCAAACTGGTGCTCAAGG (forward); CAGCCACAAAGATGGTCA (reverse); glyceraldehyde 3-phosphate dehydrogenase (GAPDH), ACTTTTAGGCGTGGCTGATG (forward);

\section{TTTTGCTGCTCACTGTATTTTATTTT}

(reverse). Level of mRNA was normalized relative to the amount of GAPDH mRNA. Data were expressed as mean value \pm standard deviation (SD). Data were analyzed using Shapiro-Wilk's test, Leven's test, one-way ANOVA and Tukey's post hoc test. All statistical analyses were performed in IBM SPSS Statistics 23 at significance level of 0.05 .

\section{RESULTS}

The combination of resveratrol and continuous exercise caused a significant increase in PDK4 expression compared with the other groups. PDK4 mRNA level in the resveratrol group and the continuous exercise group was significantly higher than in the diabetic control and sham groups (Figure 1). More importantly, the increase in the PDK4 mRNA level after combined resveratrol supplementation and continuous exercise was more profound compared to that after resveratrol and exercise alone $(\mathrm{P}<0.001)$. PDK4 mRNA levels did not differ significantly between the resveratrol and the control groups (Figure 1).

\footnotetext{
Figure 1- Comparison of the mean PDK4 mRNA levels detected by RT-PCR. There was no significant difference in the PDK4 mRNA levels between the groups with similar symbols. One-way ANOVA with post hoc-Tukey test was applied to compare mean value of parameters between all groups. C: control; DM: diabetes mellitus; CE+Resv: continuous exercise + resveratrol; CE: continuous exercise; Resv: resveratrol
}

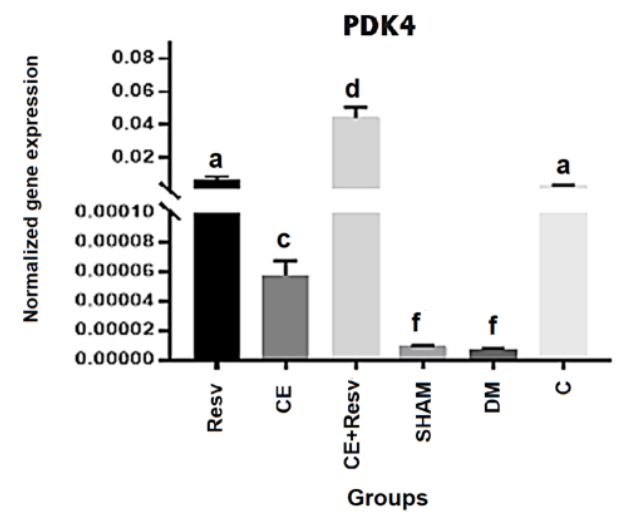


We observed no significant differences in the mean value of HOMA-IR between the study groups $(\mathrm{P}>0.05)$. In diabetics, continuous exercise, resveratrol and their combination caused a $10.9 \%, \quad 13.8 \%$ and $15.1 \%$ decrease in HOMA-IR value, respectively, compared with the control and sham groups (Figure 2).

Figure 2- Mean HOMA-IR values in the study groups. There was no significant difference in mean level of HOMA-IR between groups. C: control; DM: diabetes mellitus; CE+Resv: continuous exercise + resveratrol; CE: continuous exercise: Resv: resveratrol

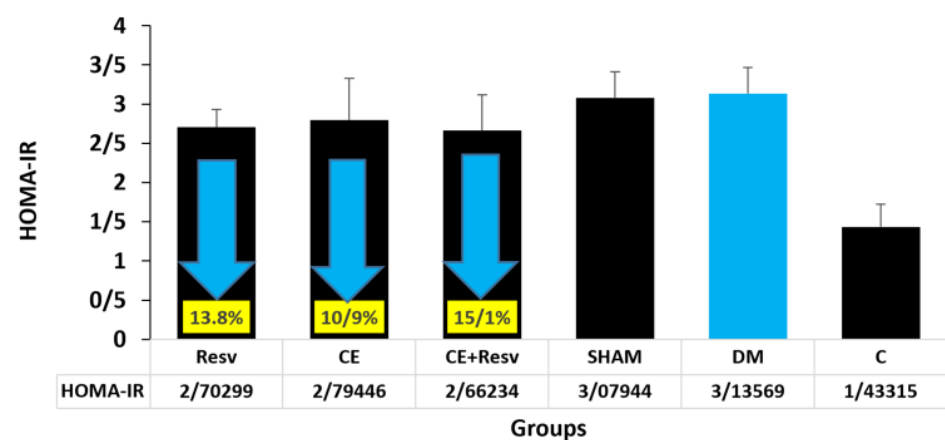

\section{DISCUSSION}

We evaluated the effects of resveratrol supplementation, continuous exercise and their combination on PDK4 expression and insulin resistance in the soleus muscle of elderly rats with type 2 diabetes. Our results indicated that the PDK4 expression is reduced in the soleus muscle of elderly rats with type 2 diabetes, which could be due to the increased PDK4 protein content in diabetic rats (32). A number of previous studies have shown that diabetes is associated with an increase in PDK4 concentration, a reduction in PDK4 gene expression, and PDK4 gene dysfunction (7, 9, 33). PDKs are regulated by insulin, glucocorticoids, thyroid hormone and fatty acids. Emerging evidence suggests that PDK decreases pyruvate dehydrogenase complex activity, which is observed in several metabolic disorders including diabetes $(7,9)$. Insulin can regulate pyruvate dehydrogenase A activity, increase pyruvate dehyrogenase phosphatase activity and reduce PDK4 level in skeletal muscles of rats (2). This suggests that PDK4-induced inhibition of pyruvate dehydrogenase and glucose oxidation inhibition may contribute to insulin resistance (2). Recent finding demonstrate that aging is associated with reduced insulin-mediated GLUT4 translocation that may contribute to insulin resistance in elderly subjects (2). In the present study, downexpression of the PDK4 gene was associated with elevated insulin resistance in the diabetic rats. It has been suggested that the PDK4 transcriptional regulation could be a potential therapeutic strategy for diabetes $(10,34)$.
In the present study, PDK4 expression increased significantly and insulin resistance decreased slightly after resveratrol supplementation in the elderly rats with type 2 diabetes. Previous studies also reported that resveratrol supplementation can improve muscle aging and insulin resistance $(19,26)$. Accumulating body of evidence shows that resveratrol activates the longevity-related protein Sirtuin 1 (SIRT1) and increases insulin sensitivity (26). Such benefits can be due to the ability of resveratrol to stimulate intracellular metabolic pathways, which improves insulin sensitivity and muscle glucose uptake, thus ultimately decreasing blood glucose levels (19). Previous studies reported that resveratrol supplementation can induce PDK4 transcription in gastrocnemius muscle in response to reduced PDK4 content (26).

We observed that PDK4 mRNA level was higher in the resveratrol and continuous exercise groups compared to the control and sham groups. More importantly, the increase in the PDK4 mRNA levels after a combination of resveratrol and continuous exercise was more profound compared to that after resveratrol and exercise alone. Resveratrol and continuous exercise induced a non-significant decrease in insulin resistance compared to treatment with resveratrol or continuous exercise alone. Several studies evaluated the effects of resveratrol supplementation along with different trainings on skeletal muscle glucose metabolism in elderly type 2 diabetic patients (2). A more recent study has shown 
that exercise, resveratrol supplementation and their combination have protective effects against sarcopenia, an age-related syndrome, by increasing the expression of p-AMPK and SIRT1 (35). The modulating effects of SIRT1 on energy homeostasis in skeletal muscles may require an upstream activating signal mediated through the energy sensor AMPK in response to resveratrol stimulation (26). Also, increased energy demand related to exercise results in increased PGC- $1 \alpha$ expression in the muscle, which in turn induces ERR $\alpha$ expression. ERR $\alpha$ then binds to the PDK4 gene promoter and induces PDK4 expression (7). Wang et al. showed that PGC$1 \alpha$ and PDK 4 mRNA levels increase by 5 to 8 fold in both muscle fiber types after exercise (14).

Although resveratrol has anti-oxidant and antiinflammatory properties, its combination with continuous exercise can be more effective for reversing the type 2 diabetes - induced

\section{REFERENCES}

1. Titus T, Badet L, Gray DW. Islet cell transplantation for insulin-dependant diabetes mellitus: perspectives from the present and prospects for the future. Expert Rev Mol Med. 2000; 2(6):1-28. doi: $10.1017 / \mathrm{S} 1462399400001861$.

2. Biensø RS, Olesen J, Gliemann L, Schmidt JF, Matzen MS, Wojtaszewski JF, et al. Effects of exercise training on regulation of skeletal muscle glucose metabolism in elderly men. Journals of Gerontology Series A: Biomedical Sciences and Medical Sciences. 2015; 70(7): 866-72.

3. Amra CA, Mette PS, Lise H, Torben H, Oluf P, Gerrit $\mathrm{vH}$, et al. The T-Allele of TCF7L2 rs7903146 associates with a reduced compensation of insulin secretion for insulin resistance induced by 9 days of bed rest. Diabetes. 2010; 59:836-43. doi: 10.2337/db09-0918.

4. Zierath JR, Krook A, Wallberg-Henriksson H. Insulin action and insulin resistance in human skeletal muscle. Diabetologia. 2000; 43(7): 821-35.

5. Snowling NJ, Hopkins WG. Effects of different modes of exercise training on glucose control and risk factors for complications in type 2 diabetic patients. Diabetes Care. 2006; 29(11): 2518-27.

6. Colberg SR, Sigal RJ, Fernhall B, Regensteiner JG, Blissmer BJ, Rubin RR, et al. Exercise and type 2 diabetes the American College of Sports Medicine and the American Diabetes Association: joint position statement. Diabetes care. 2010; 33(12): e147-e67. doi: $10.2337 / \mathrm{dc} 10-9990$.

7. Wende AR, Huss JM, Schaeffer PJ, Giguère V, Kelly DP. PGC-1_Coactivates PDK4 Gene Expression via the Orphan Nuclear Receptor ERR: a Mechanism for Transcriptional Control of Muscle Glucose Metabolism. Molecular and Cellular Biology. 2005; 25(24): 1068410694. abnormalities. It should be mentioned that the small number of rats in the subgroups is a limitation of our study.

\section{CONCLUSION}

Our results indicate that the combination of resveratrol supplementation and continuous exercise can have beneficial effects on PDK4 expression in the soleus muscle of elderly rats with type 2 diabetes. This type of intervention can also reduce insulin resistance in diabetic rats, thereby reducing the risk of diabetic complications.

\section{ACKNOWLEDGEMENTS}

We would like to thank the staff of the exercise physiology center of Islamic Azad University of Sari.

\section{CONFLICT OF INTEREST}

All authors declare that there is no conflict of interest.

8. Psilander Niklas, Wang $\mathrm{Li}$, Westergren Jens, Tonkonogi Michail, Sahlin Kent. Mitochondrial gene expression in elite cyclists: effects of high-intensity interval exercise. Eur J Appl Physiol. 2010; 110(3): 597606. doi: 10.1007/s00421-010-1544-1.

9. In-Kyu Lee. The Role of Pyruvate Dehydrogenase Kinase in Diabetes and Obesity. Diabetes Metab J. 2014; 38(3): 181-186. doi: 10.4093/dmj.2014.38.3.181.

10. Nam Ho Jeoung. Pyruvate Dehydrogenase Kinases: Therapeutic Targets for Diabetes and Cancers. Diabetes Metab J. 2015; 39(3):188-197.

11. Wang L, Sahlin K. The effect of continuous and interval exercise on PGC- $1 \alpha$ and PDK4 $m R N A$ in type I and type II fibres of human skeletal muscle. Acta physiologica. 2012; 204(4):525-32. doi: 10.1111/j.17481716.2011.02354.x.

12. Bowker-Kinley MM, Davis WI, Wu P, Harris RA, Popov KM. Evidence for existence of tissue-specific regulation of the mammalian pyruvate dehydrogenase complex. Biochem J. 1998; 329(1): 191-6. doi: 10.1042/bj3290191.

13. Zhang S, Hulver MW, McMillan RP, Cline MA, Gilbert ER. The pivotal role of pyruvate dehydrogenase kinases in metabolic flexibility. Nutr Metab (Lond). 2014; 11(1): 10. doi: 10.1186/1743-7075-11-10.

14. Wang L, Mascher H, Psilander N, Blomstrand E, Sahlin K. Resistance exercise enhances the molecular signaling of mitochondrial biogenesis induced by endurance exercise in human skeletal muscle. J Appl Physiol (1985). 2011; 111(5): 1335-44. doi: 10.1152/japplphysiol.00086.2011.

15. Stewart KJ. Exercise training: can it improve cardiovascular health in patients with type 2 diabetes? $\mathrm{Br}$ J Sports Med. 2004; 38: 250-2. doi: 10.1136/bjsm.2004.012187. 
16. Xia Q, Chen ZX, Wang YC, Ma YS, Zhang F, Che $\mathrm{W}$, et al. Association between the melatonin receptor $1 \mathrm{~b}$ gene polymorphism on the risk of type 2 diabetes, impaired glucose regulation: a meta-analysis. PLoS ONE. 2012; 7: e50107. doi: 10.1371/journal.pone.0050 107.

17. Alam S, Stolinski M, Pentecost C, Boroujerdi MA, Jones RH, Sonksen PH, et al. The effect of a six-month exercise program on very low-density lipoprotein apolipoprotein $B$ secretion in type 2 diabetes. J Clin Endocrinal Metab. 2004; 89:688-691. doi: 10.1210/jc.2003-031036.

18. Arora E, Shenoy S, Sandhu JS. Effects of resistance training on metabolic profile of adults with type 2 diabetes. Indian J Med Res. 2009; 129: 515-9.

19. Goh KP, Lee HY, Lau DP, Supaat W, Chan YH, Koh $\mathrm{AF}$. Effects of resveratrol in patients with type 2 diabetes mellitus on skeletal muscle SIRT1 expression and energy expenditure. International journal of sport nutrition and exercise metabolism. 2014; 24(1): 2-13.

20. Minakawa M, Kawano A, Miura Y, Yagasaki K. Hypoglycemic effect of resveratrol in type 2 diabetic model $d b / d b$ mice and its actions in cultured L6 myotubes and RIN-5F pancreatic $\beta$-cells. Journal of clinical biochemistry and nutrition. 2011; 48(3): 237-44.

21. Brown VA, Patel KR, Viskaduraki M, Crowell JA, Perloff M, Booth T, et al. Repeat dose study of the cancer chemopreventive agent resveratrol in healthy volunteers: safety, pharmacokinetics, and effect on the insulin-like growth factor axis. Cancer Res. 2010; 70(22): 9003-11. doi: 10.1158/0008-5472.CAN-10-2364.

22. Shang J, Chen LL, Xiao FX, Sun H, Ding HC, Xiao $\mathrm{H}$. Resveratrol improves non-alcoholic fatty liver disease by activating AMP-activated protein kinase. Acta pharmacologica Sinica. 2008 Jun; 29(6):698- 706. doi: 10.1111/j.1745-7254.2008.00807.x.

23. Rubiolo JA, Mithieux G, Vega FV. Resveratrol protects primary rat hepatocytes against oxidative stress damage: activation of the Nrf2 transcription factor and augmented activities of antioxidant enzymes. European journal of pharmacology. 2008; 591(1-3): 66-72. doi: 10.1016/j.ejphar.2008.06.067.

24. Sinha k, Chaudhary G, Gupta Y.K. Protective effect of resveratrol against oxidative stress in middle cerebral artery occlusion model of stroke in rats. Life Sciences. 2002; 71(6): 655-665.

25. Brasnyó P, Molnár GA, Mohás M, Markó L, Laczy B, Cseh J, et al. Resveratrol improves insulin sensitivity, reduces oxidative stress and activates the Akt pathway in type 2 diabetic patients. Br J Nutr. 2011; 106: 383-389. doi: 10.1017/S0007114511000316.
26. Sin TK, Yung BY, Siu PM. Modulation of SIRT1Foxol Signaling axis by Resveratrol: Implications in Skeletal Muscle Aging and Insulin Resistance. Cell Physiol Biochem. 2015; 35(2): 541-52. doi: 10.1159/000369718.

27. Batacan Jr RB, Duncan MJ, Dalbo VJ, Connolly KJ, Fenning AS. Light-intensity and high-intensity interval training improve cardiometabolic health in rats. Appl Physiol Nutr Metab. 2016; 41(9): 945-52. doi: 10.1139/apnm-2016-0037.

28. Freitas DA, Rocha-Vieira E, Soares BA, Nonato LF, Fonseca SR, Martins JB, et al.

High intensity interval training modulates hippocampal oxidative stress, BDNF and inflammatory mediators in rats. Physiol Behav. 2018; 184: 6-11. doi: 10.1016/j.physbeh.2017.10.027.

29. Linden MA, Fletcher JA, Morris EM, Meers GM, Laughlin $\mathrm{MH}$, Booth FW, et al. Treating NAFLD in OLETF rats with vigorous-intensity interval exercise training. Med Sci Sports Exerc. 2015; 47(3):556-67. doi: 10.1249/MSS.0000000000000430.

30. Mozafari M, Pharm D, Nekooeian AA, Panjeshahin MR, Zare HR. The Effects of Resveratrol in Rats with Simultaneous Type 2 Diabetes and Renal Hypertension: a Study of Antihypertensive Mechanism. Iran J Med Sci March. 2015; 40(2):152-160.

31. Delaviz H, Davoudi P, Sharif Talebian Pour M, Rad $\mathrm{P}$, Sadeghi $\mathrm{H}$. The effect of resveratrol on fetal heart tissue changes after preeclampsia induced by l-name in rats. J Shahid Sadoughi Univ Med Sci. 2015; 23(7): 66978.

32. Aminizadeh $\mathrm{S}$, Habibie A, Marefati $\mathrm{H}$, Shakerian $\mathrm{S}$. The Role of Pyruvate Dehydrogenase Kinase 4 (PDK4) on the Expression of Citrate Synthase in the Skeletal Muscle After 4 Weeks of Endurance Training in Male Wistar Rats. Journal of Rafsanjan University of Medical Sciences. 2017; 16(3):191-202.

33. Tao R, Xiong X, Harris RA, White MF, Dong XC. Genetic inactivation of pyruvate dehydrogenase kinases improves hepatic insulin resistance induced diabetes. PloS one. 2013; 8(8):e71997.

34. Dlamini Z, Ntlabati P, Mbita Z, Shoba-Zikhali L. Pyruvate dehydrogenase kinase 4 (PDK4) could be involved in a regulatory role in apoptosis and a link between apoptosis and insulin resistance. Exp Mol Pathol. 2015; 98(3): 574-84. doi: 10.1016/j.yexmp.2015.03.022.

35. Liao ZY, Chen JL, Xiao MH, Sun Y, Zhao YX, Pu $\mathrm{D}$, et al. The effect of exercise, resveratrol or their combination on Sarcopenia in aged rats via regulation of AMPK/Sirt1 pathway. Exp Gerontol. 2017; 98:177-183. doi: 10.1016/j.exger.2017.08.032. 